\title{
Biomarkers Predict Relapse in Granulomatosis with Polyangiitis
}

\author{
Patrick C. P. Hogan, ${ }^{1}$ Robert M. O'Connell, ${ }^{1}$ Simone Scollard, ${ }^{1,2}$ Emmett Browne, ${ }^{1}$ \\ Emer E. Hackett, ${ }^{1}$ and Conleth Feighery ${ }^{1}$ \\ ${ }^{1}$ Department of Immunology, Trinity College Dublin, St. James's Hospital, Dublin 8, Ireland \\ ${ }^{2}$ Institute of Molecular Medicine, Trinity College Dublin, St. James's Hospital, Dublin 8, Ireland \\ Correspondence should be addressed to Conleth Feighery; con.feighery@tcd.ie
}

Received 31 October 2013; Revised 21 March 2014; Accepted 5 April 2014; Published 30 April 2014

Academic Editor: Philip Thomas

Copyright (C) 2014 Patrick C. P. Hogan et al. This is an open access article distributed under the Creative Commons Attribution License, which permits unrestricted use, distribution, and reproduction in any medium, provided the original work is properly cited.

\begin{abstract}
Granulomatosis with polyangiitis (GPA) is a small blood vessel vasculitic disorder with a high mortality rate if undiagnosed or treated inadequately. Disease relapse is a key feature of this disease and early identification of relapse episodes is very important in limiting end-organ damage. The advent of indirect immunofluorescence to detect antineutrophil cytoplasmic antibody (ANCA) with specific reactivity against the enzyme proteinase-3 (PR3) has been very useful in the diagnosis of GPA but is less helpful in predicting relapse. Indeed, up to date no satisfactory biomarker has been identified that can reliably predict relapse. This study assessed the probability of the occurrence of a relapse when a change was noted in a range of commonly used laboratory tests. These tests included levels of C-reactive protein (CRP), anti-PR3 antibodies, ANCA titre, and the neutrophil count. A group of 30 GPA patients with a total of 66 relapse episodes was investigated and a novel clinical yield score was devised. When a combined rise in CRP, anti-PR3 antibodies, and neutrophil count was observed in the 6-month period before a relapse event, 59\% of patient relapses could be predicted. Monitoring changes in this set of parameters helps identify disease relapse.
\end{abstract}

\section{Introduction}

Granulomatosis with polyangiitis (GPA) is an autoimmune vasculitis primarily affecting the respiratory tract and kidneys and is characterised by necrosis and granulomatous lesions involving small to medium sized blood vessels. The principal autoantibody associated with the disease is the antineutrophil cytoplasmic antibody (ANCA), usually directed against the enzyme proteinase-3 (PR3) [1, 2].

Long term survival in GPA has greatly improved since the introduction of disease modifying agents such as cyclophosphamide, with a ten-year survival of approximately $75 \%$ [3]. Disease relapse is a major cause of morbidity and mortality in patients with GPA, with some $50 \%$ of patients experiencing relapse at five-year followup [4]. Prediction of disease relapse is therefore an important goal of clinical management. Certain patient and disease factors have been identified as increasing the risk of relapse. For example, it has been shown that lung involvement, infection with staphylococcus aureus, and cardiac or renal involvement increase the chance of relapse [5-7].

The identification of biomarkers that predict relapse in GPA has proven elusive. While a change in ANCA titre may be associated with disease activity, it is not sufficiently specific nor sensitive to be an effective predictor of relapse [8] and hence is considered to be no more than moderately useful $[9,10]$. For example, if a rise in ANCA titre alone was used as an indicator of disease relapse, this could expose patients to unnecessary and potentially toxic effects of immunosuppression [11]. No role in the prediction of relapse has been found for conventionally measured inflammatory markers such as $\mathrm{C}$-reactive protein (CRP) or the erythrocyte sedimentation rate [12].

The purpose of this study was to examine whether a combination of commonly measured laboratory parameters might help predict disease relapse in patients withv GPA. A novel clinical yield formula was devised to examine changes 
in the identified biomarkers in the six-month period before the relapse event.

\section{Methods}

Patients attending St. James's Hospital who had been diagnosed with GPA between 1984 and 2012 were included. The study was conducted using patients' medical charts and the hospital investigation database. Patients were required to have had evidence of one or more major relapse events to be eligible for inclusion. A major relapse was defined as the commencement of potent immunosuppressive therapy, such as cyclophosphamide or rituximab, together with high dose prednisolone. Disease relapse was distinguished from an intercurrent infection based on the clinical judgment of a consultant clinical immunologist. Patients were also required to have a granular, cytoplasmic staining pattern on ANCA indirect immunofluorescence (IIF) and to be anti-PR3 antibody positive on enzyme-linked immunosorbent assay (ELISA). Test results for eight biomarkers were collected for each patient throughout followup: ANCA titre, anti-PR3 antibody level, CRP and creatinine levels, white cell count, neutrophil count, lymphocyte count, and monocyte count.

For ANCA IIF, serum samples were diluted 1/20 and examined using INOVA neutrophil coated slides. Anti-PR3 antibody levels were measured using the Biodiagnostics ELISA system. Full blood and differential white cell counts were performed on automated equipment (Sysmex XE2000) and creatinine levels assayed using the Hitachi-747 analyser. CRP values were measured using the Behring BN2 nephelometer.

For each biomarker, a mean remission baseline value was calculated in order to exclude results which coincided with periods of disease activity. Hence, the following test results were excluded in calculating this remission baseline: all results from the twelve-month period after diagnosis and results from the six-month period before and the six-month period after the occurrence of a relapse. Having excluded these results, the remaining test results were termed as the remission baseline value. The baseline values were then used to calculate a "clinical yield" according to the following formula, in which the "prerelapse period" is defined as the six-month period prior to relapse:

Clinical Yield $=(($ Number of Results (elevated or decreased) in pre-relapse period)/(Number of Results (elevated or decreased) in remission and pre-relapse period)) $\times 100$.

The clinical yield formula has been previously used to evaluate the usefulness of other diagnostic techniques [13]. The value of this method of analysis is that it gives a true representation of the usefulness of tests, since it includes all the patients' test results as they were carried out in the clinical setting. Statistical significance was calculated using the Student's $t$-test.

Statistically significant results were combined to produce a composite clinical yield value for concurrent elevations in these biomarkers. White cell count was omitted from this
TABLE 1: Clinical details of 30 patients with GPA with major disease relapse events.

\begin{tabular}{lc}
\hline Characteristic & Cohort \\
\hline Total number of patients & 30 \\
Male & $15(50 \%)$ \\
Female & $15(50 \%)$ \\
Mean age at diagnosis (years) & 47.57 ( \pm 16 years) \\
Mean length of followup (years) & 11.73 ( \pm 5 years) \\
Mean age at first relapse (years) & 52.1 ( \pm 16 years) \\
Mean time from diagnosis to first relapse (years) & 4.53 ( \pm 5 years) \\
Total number of relapse events & 66 \\
Mean number of relapses per patient & 2.2 \\
\hline
\end{tabular}

TABLE 2: Clinical yield of a series of relapse biomarkers in 30 patients with GPA.

\begin{tabular}{lccc}
\hline Biomarker & $\begin{array}{c}\text { Clinical yield } \\
\text { if }<\text { baseline }\end{array}$ & $\begin{array}{c}\text { Clinical yield } \\
\text { if }>\text { baseline }\end{array}$ & $P$ value \\
\hline White cell count & $11.22 \%$ & $30.21 \%$ & 0.01 \\
Neutrophil count & $11.98 \%$ & $31.43 \%$ & 0.01 \\
Lymphocyte count & $18.13 \%$ & $23.53 \%$ & 0.92 \\
Monocyte count & $20.23 \%$ & $21.40 \%$ & 0.63 \\
ANCA titre & $18.24 \%$ & $22.79 \%$ & 0.7 \\
Anti-PR3 antibodies & $7.86 \%$ & $24.81 \%$ & 0.0003 \\
CRP & $15.67 \%$ & $32.31 \%$ & 0.002 \\
Creatinine & $17.33 \%$ & $23.40 \%$ & 0.74 \\
Neutrophil count, CRP, & $14.81 \%$ & $58.89 \%$ & 0.02 \\
and anti-PR3 & & & \\
\hline
\end{tabular}

calculation to prevent duplication of results of the neutrophil count.

\section{Results and Discussion}

In total 52 patients were identified for participation in this study. Of these, 22 patients were excluded from the study; 12 had never had a relapse; and a further 10 had inadequate test results. The characteristics of the remaining cohort of 30 patients are shown in Table 1. These 30 patients experienced a total of 66 relapses events. The total number of test results from these patients was 8,376. This was composed of 716 ANCA tests; 494 anti-PR3 antibody tests; 1,212 CRP measurements; and 1,198 white cell counts with a similar number of neutrophil, monocyte, and lymphocyte counts. 1,176 creatinine tests were also evaluated. The clinical yields of these biomarkers are shown in Table 2.

The clinical yield for all the biomarkers was greater for elevated results in comparison to decreased levels. For example, the results show a clinical yield of $32.31 \%$ for an elevation in CRP when compared to baseline values and $15.67 \%$ for a decrease in CRP. This means that the finding of an elevation in the CRP level was followed by a relapse within 6 month in $32.31 \%$ of cases but not in the remaining $67.69 \%$ of such cases. Likewise, when CRP values were lower than 
baseline values, this decrease preceded a relapse in $15.67 \%$ of cases.

The clinical yield for changes in white cell count, neutrophil count, CRP, and anti-PR3 antibody values was each statistically significant (Table 2). When the results for the neutrophil count, CRP, and anti-PR3 antibodies were examined as a group, the clinical yield was found to be $58.89 \%$ $(P=0.02)$. Thus, from a clinical perspective, a relapse could be predicted to develop within 6 months in some 59\% of such cases. This finding matches clinical experience; for example, when a patient demonstrates an evolving rise in CRP, antiPR3 antibodies, and neutrophil count, this is frequently accompanied by clinical deterioration and eventual relapse. Nonetheless, reliance on these three parameters would fail to indicate the development of relapse in $41 \%$ of cases. Further information can be gleaned from the situation where there is a fall in the levels of these parameters, when relapse is very unlikely and in $85 \%$ of such cases, patients are predicted to remain relapse-free. Finally, the results showed no significant predictive value for ANCA titres, serum creatinine levels, monocyte counts, or lymphocyte counts, when examined alone (Table 2).

\section{Conclusions}

After clinical remission has been induced in a patient, the common practice is to reduce and if possible to stop immunosuppressive treatment entirely. However, because disease relapse is so common, developing in $50 \%$ of patients [4], it is important to identify relapse early, so further treatment with immunosuppression can be introduced [14, 15]. However, clinicians are very mindful of the potential toxic side effects of this therapy [16]. On occasions, secure identification of a relapse is difficult. Hence, clinical details and laboratory results are carefully evaluated to determine if a relapse is taking place. Numerous studies have assessed the usefulness of ANCA in relapse prediction in GPA. A recent meta-analysis of such trials showed a link between a rise or persistent elevation in ANCA and relapse. However, the authors of this meta-analysis acknowledge that their results show only a modest correlation [9]. The findings of the present study show that when a rise is observed in the combination of the neutrophil count, CRP levels, and antiPR3 antibodies, this is useful adjunctive evidence of relapse. This combination was found to be more strongly associated with relapse than any biomarker alone. However, there is still a need to develop improved biomarkers of disease activity in this life-threatening inflammatory disorder.

\section{Disclosure}

This study was presented at the 16th International vasculitis and ANCA workshop, Paris 2013, and the Irish Association of Allergy and Immunology, Leixlip, 2012.

\section{Conflict of Interests}

The authors declare that there is no conflict of interests regarding the publication of this paper.

\section{Acknowledgments}

Caroline Liddy provided excellent general support for the study and in particular in helping source the laboratory data on the patients.

\section{References}

[1] F. J. van der Woude, N. Rasmussen, S. Lobatto et al., "Autoantibodies against neutrophils and monocytes: tool for diagnosis and marker of disease activity in Wegener's granulomatosis," The Lancet, vol. 1, no. 8426, pp. 425-429, 1985.

[2] J. C. Jennette, J. R. Hoidal, and R. J. Falk, "Specificity of antineutrophil cytoplasmic autoantibodies for proteinase 3," Blood, vol. 75, no. 11, pp. 2263-2264, 1990.

[3] W. Koldingsnes and H. Nossent, "Predictors of survival and organ damage in Wegener's granulomatosis," Rheumatology, vol. 41, no. 5, pp. 572-581, 2002.

[4] C. P.-D. Despujol, J. Pouchot, C. Pagnoux, J. Coste, and L. Guillevin, "Predictors at diagnosis of a first Wegener's granulomatosis relapse after obtaining complete remission," Rheumatology, vol. 49, no. 11, Article ID keq244, pp. 2181-2190, 2010.

[5] C. Pagnoux, S. L. Hogan, H. Chin et al., "Predictors of treatment resistance and relapse in antineutrophil cytoplasmic antibodyassociated small-vessel vasculitis," Arthritis and Rheumatism, vol. 58, no. 9, pp. 2908-2918, 2008.

[6] C. A. Stegeman, J. W. C. Tervaert, P. E. De Jong, and C. G. M. Kallenberg, "Trimethoprim-sulfamethoxazole (Cotrimoxazole) for the prevention of relapses of Wegener's granulomatosis," New England Journal of Medicine, vol. 335, no. 1, pp. 16-20, 1996.

[7] C. Mukhtyar, O. Flossmann, B. Hellmich et al., "Outcomes from studies of antineutrophil cytoplasm antibody associated vasculitis: a systematic review by the European League Against Rheumatism systemic vasculitis task force," Annals of the Rheumatic Diseases, vol. 67, no. 7, pp. 1004-1010, 2008.

[8] N. Rasmussen, A. Salmela, A. Ekstrand et al., "Changes in proteinase 3 anti-neutrophil cytoplasm autoantibody levels in early systemic granulomatosis with polyangiitis (Wegener's) may reflect treatment rather than disease activity," Clinical and Experimental Rheumatology, vol. 31, no. 1, supplement 75, pp. S38-S44, 2013.

[9] G. Tomasson, P. C. Grayson, A. D. Mahr, M. LaValley, and P. A. Merkel, "Value of ANCA measurements during remission to predict a relapse of ANCA-associated vasculitis-a metaanalysis," Rheumatology, vol. 51, no. 1, pp. 100-109, 2012.

[10] C. A. Staegeman, "Anti-neutrophil cytoplasmic antibody (ANCA) levels directed against proteinase-3 and myelop-er-ox$\mathrm{i}$-das-e are helpful in predicting disease relapse in ANCAassociated small-vessel vasculitis," Nephrology Dialysis Transplantation, vol. 17, no. 12, pp. 2077-2080, 2002.

[11] X. Bosch, A. Guilabert, and J. Font, "Antineutrophil cytoplasmic antibodies," The Lancet, vol. 368, no. 9533, pp. 404-418, 2006.

[12] A. I. Kälsch, E. Csernok, D. Münch et al., "Use of highly sensitive C-reactive protein for followup of Wegener's granulomatosis," Journal of Rheumatology, vol. 37, no. 11, pp. 2319-2325, 2010.

[13] L. A. Hirano, S. T. Bogardus Jr., S. Saluja, L. Leo-Summers, and S. K. Inouye, "Clinical yield of computed tomography brain scans in older general medical patients," Journal of the American Geriatrics Society, vol. 54, no. 4, pp. 587-592, 2006. 
[14] C. Mukhtyar, L. Guillevin, M. C. Cid et al., "EULAR recommendations for the management of primary small and medium vessel vasculitis," Annals of the Rheumatic Diseases, vol. 68, no. 3, pp. 310-317, 2009.

[15] C. A. Langford, "Update on the treatment of granulomatosis with polyangiitis (Wegener's)," Current Treatment Options in Cardiovascular Medicine, vol. 14, no. 2, pp. 164-176, 2012.

[16] G. S. Hoffman, G. S. Kerr, R. Y. Leavitt et al., "Wegener gran-ulomatosis: an analysis of 158 patients," Annals of Internal Medicine, vol. 116, no. 6, pp. 488-498, 1992. 


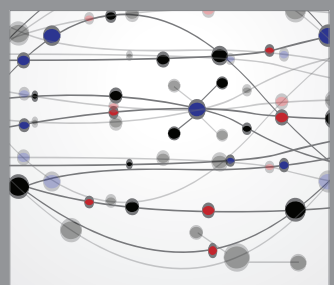

The Scientific World Journal
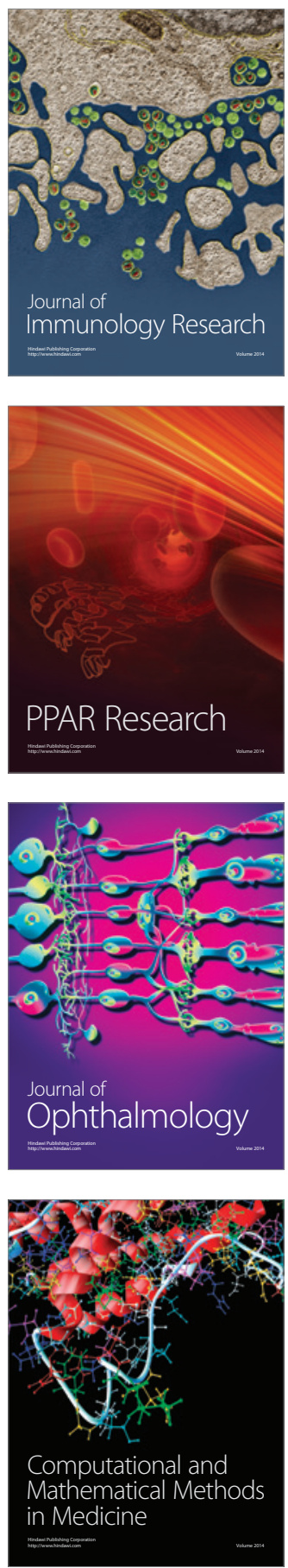

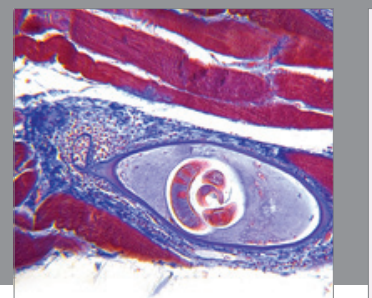

Gastroenterology

Research and Practice
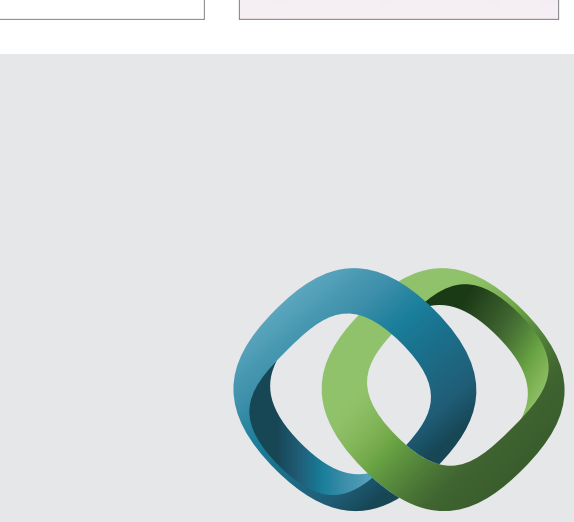

\section{Hindawi}

Submit your manuscripts at

http://www.hindawi.com
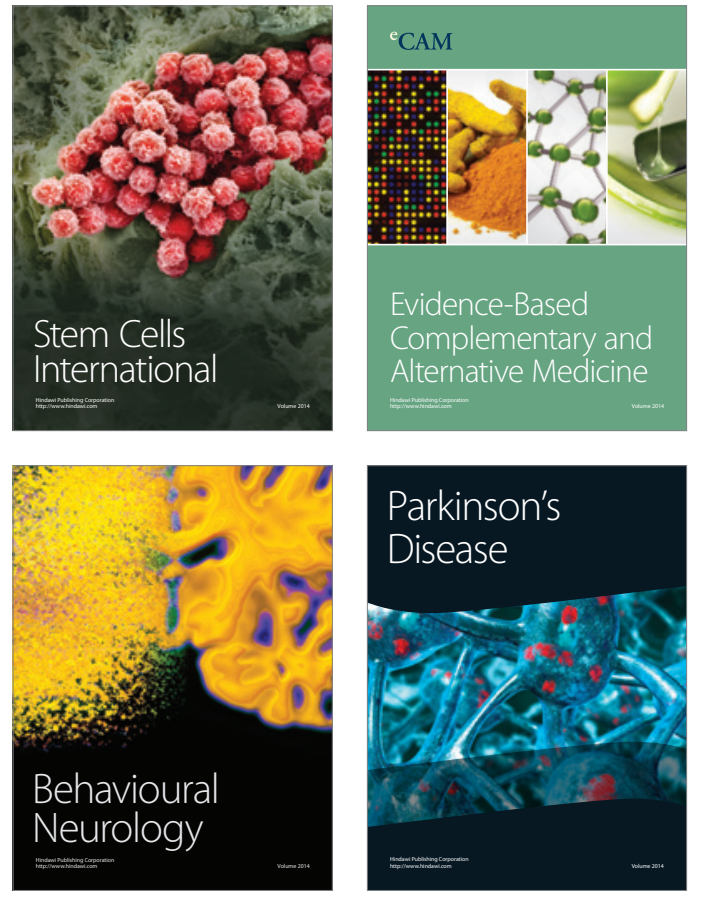
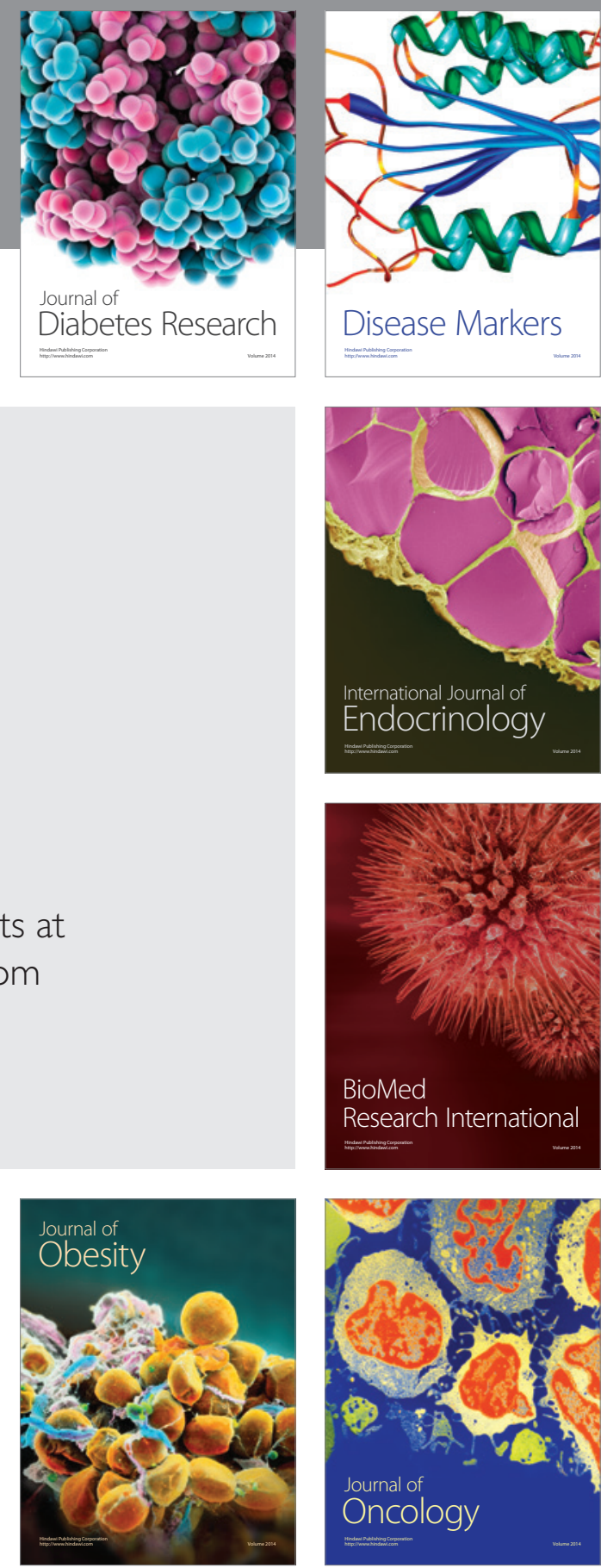

Disease Markers
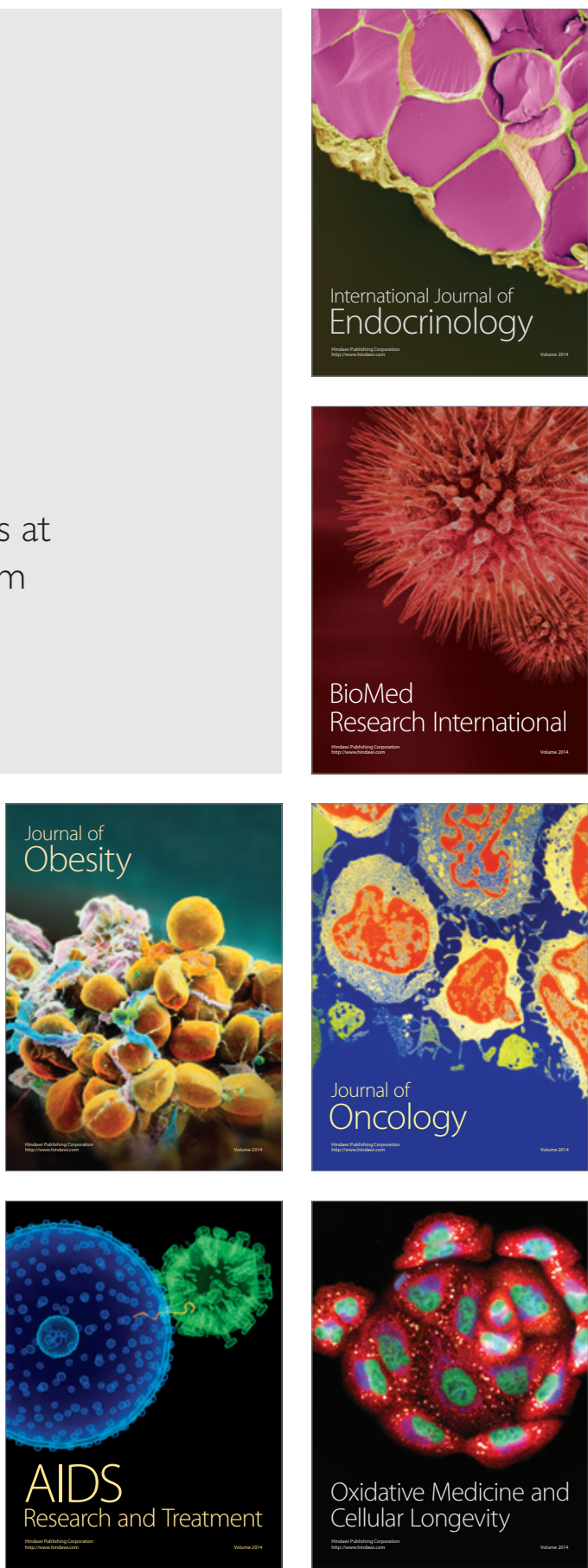